\title{
Research Paper \\ Reliability of Functional Balance Tests and Their Correlation With Selected An- thropometric Parameters in Children Aged 7-10 Years
}

\author{
*Mahboobeh Dehnavi ${ }^{1}$ (1) Heidar Sadeghi $^{1}$, Mehdi Taghva ${ }^{1}$ \\ 1. Department of Sports Biomechanics, Faculty of Physical Education and Sports Science, Central Tehran Branch, Islamic Azad University, Tehran, Iran.
}

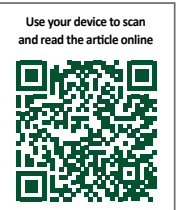

Cttation: Dehnavi M, Sadeghi H, Taghva M. [Reliability of Functional Balance Tests and Their Correlation With Selected Anthropometric Parameters in Children Aged 7-10 Years (Persian)]. Journal of Sport Biomechanics. 2020; 5(4):216-227. https:// doi.org/10.32598/biomechanics.5.4.2

dol 'https://doi.org/10.32598/biomechanics.5.4.2

Key words:

Reliability, Static

balance, Dynamic balance, Anthropometric parameters, Children

\section{ABSTRACT}

Objective The present study aimed to evaluate the reliability of functional balance tests and their correlation with selected anthropometric parameters in children aged 7-10 years.

Methods Participants were 80 students aged 7-10 (40 female and 40 male). Romberg Test and Sharpened Romberg Test were used for assessing static balance, while timed up and go test, tandem walk test, and Y-balance test were used for measuring dynamic balance. Selected anthropometric factors were body height, upper body length, lower body length, Foot length and body mass. Reliability was determined using intra-class correlation coefficient (ICC), and Pearson correlation was used for examining the relationship between balance tests and selected anthropometric parameters at a significance level of $P \geq 0.05$.

Results Static balance tests were not reliable, but dynamic tests were reliable. No significant correlation was found between anthropometric parameters and static and dynamic tests $(P>0.05)$; except between lower body length and $\mathrm{Y}$-balance test $(\mathrm{r}=0.53, \mathrm{P}=0.01)$.

Conclusion It seems that at the age of 7-10 years, static balance tests are affected because the sensory systems related to balance are developing. Therefore, static balance tests, which require weighting of each of these systems with eyes closed and open, are not good criteria for examining the balance of this age group. Hence, it is better to use dynamic balance tests, especially the Y-balance test.

\section{Extended Abstract}

\section{Introduction}

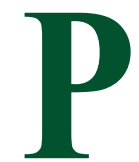

osture control involves controlling the position of the body in space for two purposes: stability and body orientation. The orientation component is defined as the ability to maintain communication between different parts of the body and also between the body and the environment to perform a specific task [1]. The sta- bility component referred to static balance, which defines the ability to maintain the center of gravity within the base of support [2] which is effective in performing many daily activities; it has been found that daily activities require certain levels of balance [3]. One of the key factors in assessing the development of children's motor skills is balance assessment [2].

Many balance tests are used to differentiate between healthy children and children with developmental disorders. According to the results of these tests, specialists inter-

* Corresponding Author:

Mahboobeh Dehnavi, MSc.

Address: Department of Sports Biomechanics, Faculty of Physical Education and Sports Science, Central Tehran Branch, Islamic Azad University Tehran, Iran.

Tel: +98 (935) 3954320

E-mail: mahboobeh.dehnavi@gmail.com 
pret, compare and make decisions about the development of children's motor skills and subsequently adopt the necessary therapeutic and training interventions [4]. Therefore, these balance tests must be reliable and accurate, because the decisions that are made for children based on the results of these balance tests are crucial. Hence, we should identify valid and appropriate tests for this group. In this regard, this study aimed to evaluate the reliability of selected functional balance tests in children aged 7-10 years, and the relationship between these functional balance tests and selected anthropometric parameters to determine the role of anthropometric parameters on the balance of this age group.

\section{Methods}

This is a descriptive/correlational study. The study population consisted of all male and female students aged 7-10 years in Sama Elementary School located in Neishabour, Iran. Using cluster sampling method, 80 students (40 girls and 40 boys) were selected as the study samples. Subjects in both groups had no any history of injury in the past year, no damage to the visual and vestibular systems, and no any neurological disorder and postural abnormalities (e.g. lordosis, flat feet, genu valgum, genu varum). After giving information about the study protocol, an informed consent was obtained from all the participants. The study was conducted in a suitable and quiet place inside the school.

First, a questionnaire of personal information, health and physical activity status was provided to the subjects to ensure that the subjects were prepared to perform balance tests. Then the measurements related to the descriptive information of the subjects and the selected anthropometric parameters were performed. Finally, static and dynamic balance tests were performed in three sessions (Table 1). To reduce the learning effect, each session was performed with a 72-h interval. Romberg test [5], and Sharpened Romberg test [6] were used to evaluate static balance and Timed Up and Go test [7], tandem walk test [8], and Y balance test [9] were used to evaluate dynamic balance in three scoring times. Body height, upper body length, lower body length, foot length and body mass were used as anthropometric factors [10].

\section{Results}

The purpose of this study was to evaluate the reliability of functional balance tests and the relationship between these tests and selected anthropometric parameters in girls and boys aged 7-10 years. The results of the present study showed that none of the static balance tests including Romberg test and Sharpened Romberg balance test had the acceptable reliability for girls and boys. Regarding the dynamic balance tests, based on the ICC value, the reliability of timed up and go test and tandem walk test were reliable only for the boys, while Y balance test had the required reliability in both boys and girls. Moreover, the results showed no significant relationship between anthropometric variables and static and dynamic balance tests except between lower body length and $\mathrm{Y}$ balance test.

\section{Discussion and Conclusion}

It seems that at the age of 7-10 years, static balance tests are affected because the sensory systems related to balance are developing. Therefore, static balance tests, which re-

Table 1. Results of static and dynamic balance tests in three sessions

\begin{tabular}{|c|c|c|c|c|c|c|}
\hline \multirow{2}{*}{ Test } & \multirow{2}{*}{ Gender } & \multicolumn{3}{|c|}{ Mean \pm SD } & \multicolumn{2}{|c|}{ Reliability } \\
\hline & & Session 1 & Session 2 & Session 3 & ICC & $\mathbf{P}$ \\
\hline \multirow{2}{*}{ Romberg test (s) } & Boy & $45.18 \pm 36.55$ & $51.14 \pm 85.10$ & $54.13 \pm 28.27$ & 0.58 & 0.12 \\
\hline & Girl & $49.16 \pm 72.06$ & $56.8 \pm 30.50$ & $55.10 \pm 57.67$ & 0.19 & 0.46 \\
\hline \multirow{2}{*}{ Sharpened Romberg test (s) } & Boy & $14.5 \pm 22.63$ & $11.6 \pm 70.74$ & $10.3 \pm 55.52$ & 0.34 & 0.26 \\
\hline & Girl & $20.4 \pm 68.32$ & $23.7 \pm 37.36$ & $18.3 \pm 85.51$ & 0.57 & 0.11 \\
\hline \multirow{2}{*}{ Timed Up and Go test (s) } & Boy & $6.0 \pm 78.9$ & $6.0 \pm 32.61$ & $6.0 \pm 57.53$ & 0.79 & $* 0.02$ \\
\hline & Girl & $4.0 \pm 9.91$ & $5.1 \pm 76.52$ & $7.1 \pm 57.67$ & 0.56 & 0.08 \\
\hline \multirow{2}{*}{ Tandem walk test (number of errors) } & Boy & $11.4 \pm 21.28$ & $12.3 \pm 70.74$ & $11.3 \pm 32.28$ & 0.75 & $* 0.02$ \\
\hline & Girl & $12.3 \pm 22.18$ & $15.6 \pm 11.37$ & $10.5 \pm 19.91$ & 0.48 & 0.12 \\
\hline \multirow{2}{*}{ Y balance test (percentage of leg length) } & Boy & $71.14 \pm 92.51$ & $73.13 \pm 47.52$ & $73.11 \pm 90.9$ & 0.81 & $* 0.01$ \\
\hline & Girl & $69.12 \pm 55.21$ & $69.11 \pm 10.87$ & $70.12 \pm 22.47$ & 0.80 & $*^{*} 0.01$ \\
\hline
\end{tabular}


quire weighting of each of these systems with eyes closed and open, are not good criteria for examining the balance of this age group. Hence, to examine, interpret and compare the results of balance in these ages, it is better to use dynamic balance tests, especially the Y balance test.

\section{Ethical Considerations}

Compliance with ethical guidelines

All ethical principles were considered in this article.

Funding

The present paper was extracted from the MSc. thesis of the first author, Department of Sports Biomechanics, Faculty of Physical Education and Sports Science, Central Tehran Branch, Islamic Azad University.

Authors' contributions

Conceptualization, Methodology, Supervision: All authors; Invesigation, Writing original draft, Funding acquisition, Resources: Mahboobeh Dehnavi; Writing-review and editing: Mahboobeh Dehnavi, Heidar Sadeghi.

Conflicts of interest

The authors declared no conflict of interest.

Acknowledgements

The authors would like to thank Javad Harati and all who helped and participated in the completion of this study. 


\title{
هايايیىسنجى أزمون هاى عملكردى تعادل در دختران و يسران Vتا + اساله وارتباط آن با هار امترهاى منتخب آنترويومتريكى أزمانى
}

\author{
"محبوبه دهنوى' هـ حيدر صادقى'، مهدى تقوا' \\ 1. تروه بيومكانيك ورزشى، دانشكده تربيتبدنى و علوم ورزشى، واحد تهران مركز، دانشعاه آزاد اسلامى، تهران، ايران.
}

\begin{abstract}
حكיد
هدف يرؤشش حاضر با هدف ياياييىسيجى آزمونهاى عملكردى تعادل و ارتباط اين آزمون ها با بارامترهاى ميتخب آنترويومتريكى در دختران و يسران هفت تأ دمساله بود.

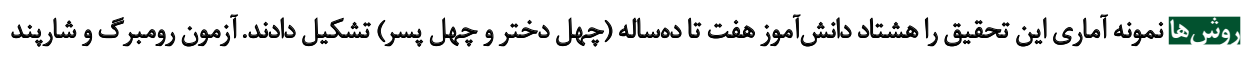

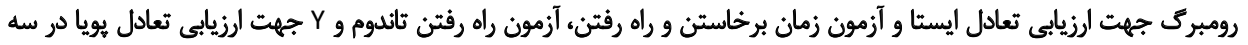

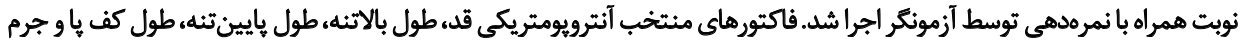

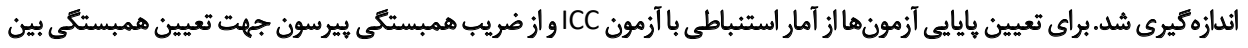

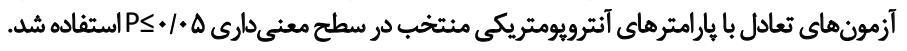

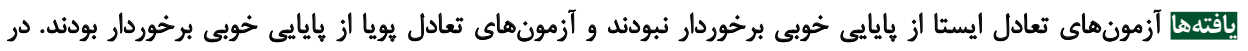

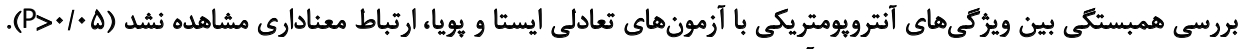

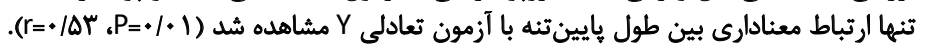

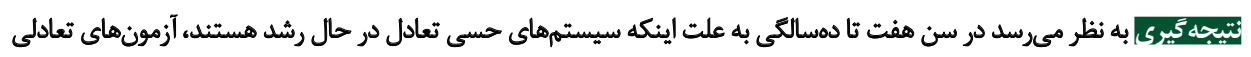

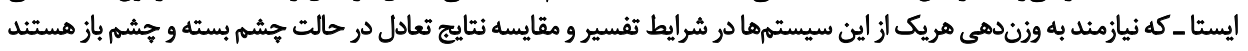

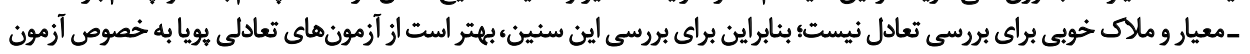

اطلاعات مقاله:

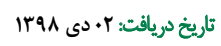

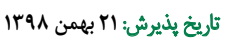

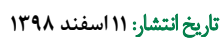

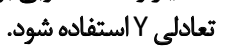

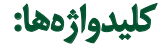

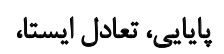

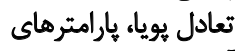
أنترويومتريكى، تعادل بارل

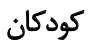

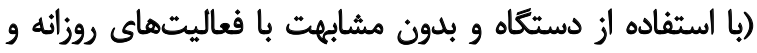

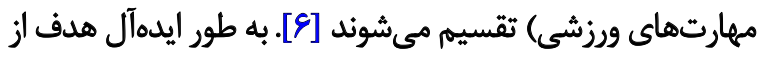

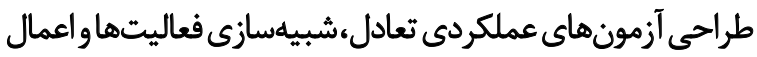

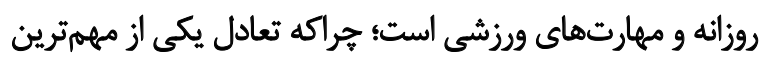

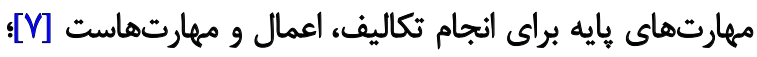

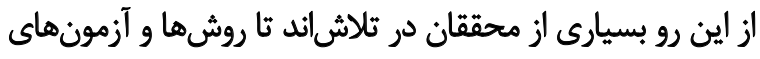

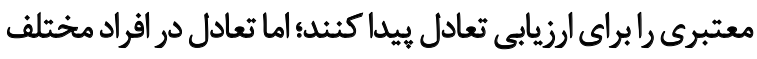

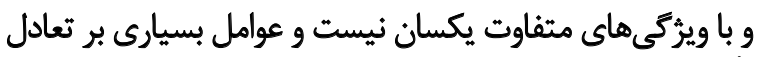

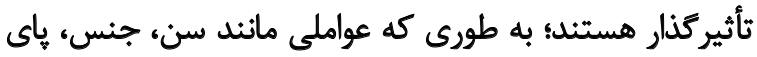

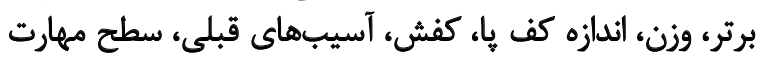

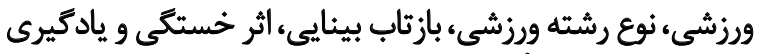

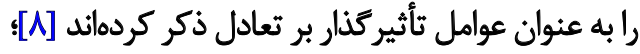
بنابراين با توجه به مطالب ارائهشده انتخاب آزمون تعادلى بردي

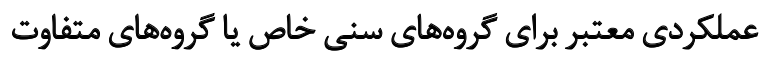

كنترل ياستحر شامل كنترل موقعيث بدن در فضا براي دو هدف

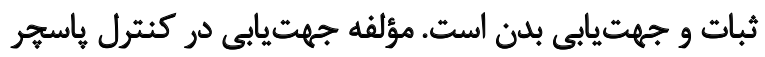

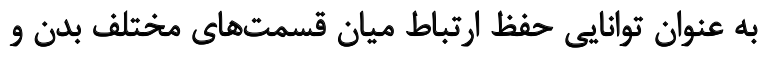

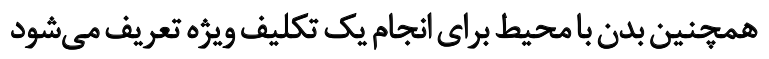

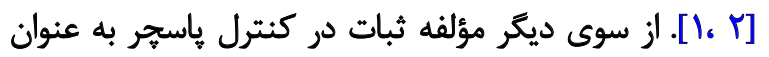

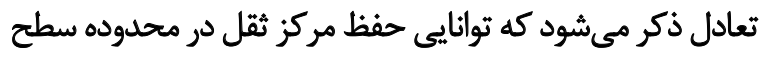

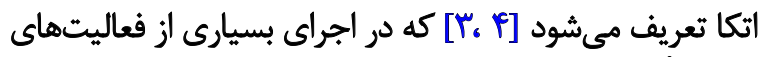

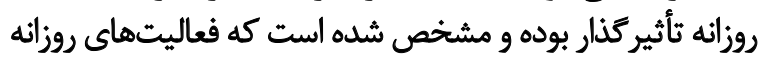

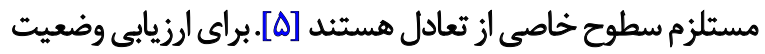
تعادل از روش هاى مختلفى استفاده مي إشود. به طور كلى آزمونهاي ارزيابي تعادل به دو دسته عملكردى

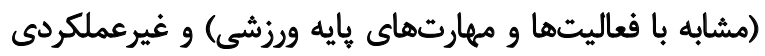

* نويسئده مسينول:

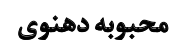
نشاني: كروه بيومكانيك ورزشي، داتشكده تربيتبدنى و علوم ورزشي، واحد تهران مركز، دانشكاه آزاد اسلامي، تهرانه ايران.

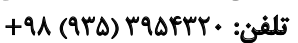
mahboobeh.dehnavi@gmail.com : يست الكترونيكي • 
مي شود [1ه]]؛ بنابراين هنكام استفاده از آزمونهاي ارزيابى تعادل

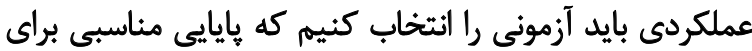

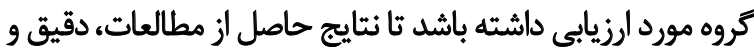

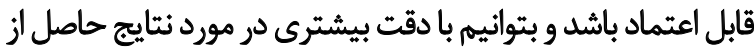

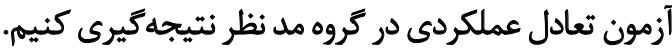
يكى از فاكتورهاى اساسى براى ارزيابى رشد مهارتهاتياي

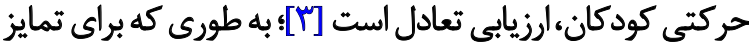

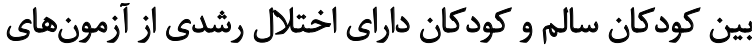

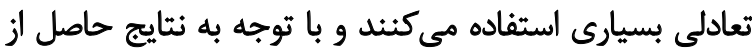

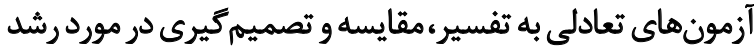

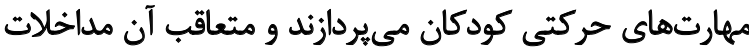

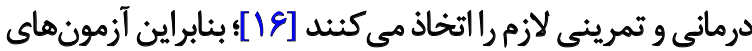

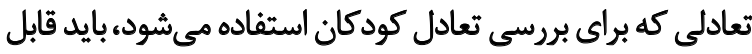

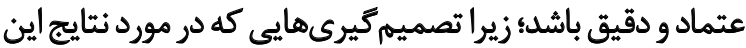

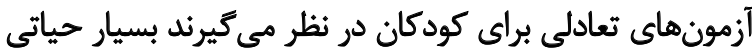

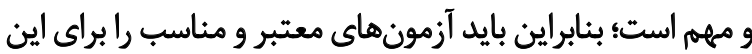

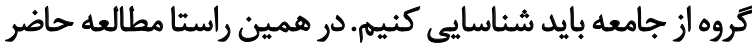

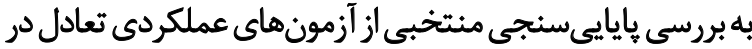

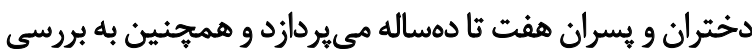

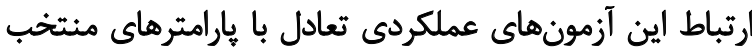

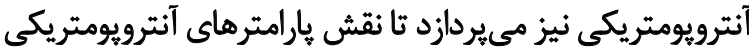

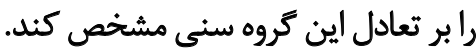

مواد ورشها

أزمودنى ها

روش اين يُروهش از نظر هدف، كاربردى و به لحاظ تردآورى

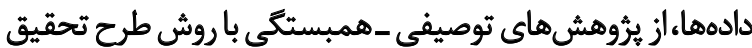

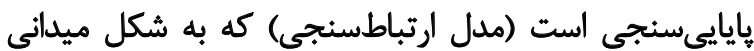

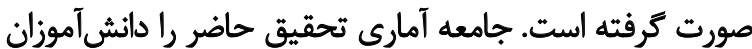

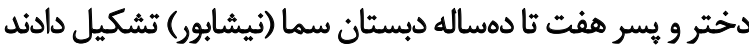

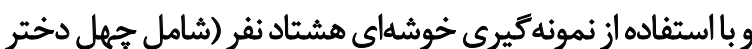

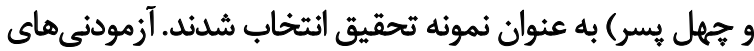

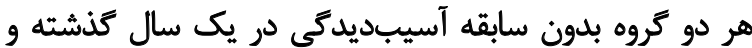

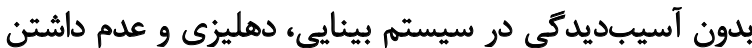

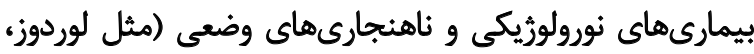

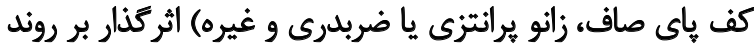

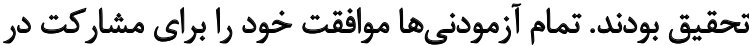

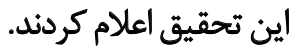

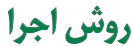

تحقيق حاضر در فضاى مناسب و آرامى كه در داخل مدرسه

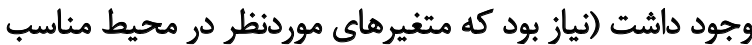

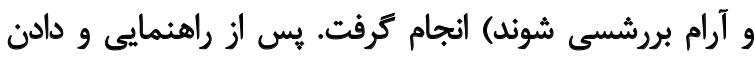

جامعه بسيار مهم است و در كروههاى مختلف ممكن است

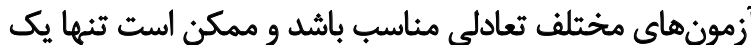

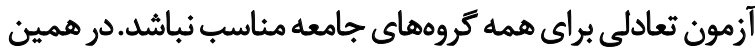

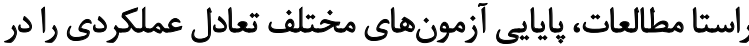
كروههاى مختلف جامعه بررسى كردهاند.

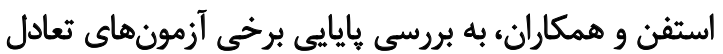

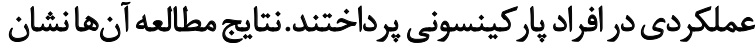

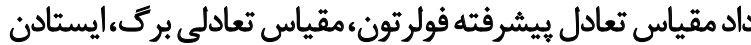

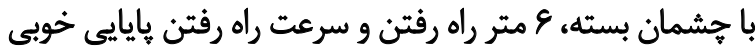

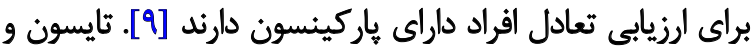

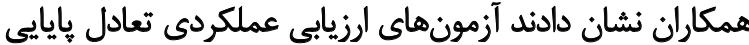

خوبى براى ارزيابى تعادل افراد سكته مغزى كرده دارئ دارند [ • 1]. بروكليو و همكاران نيز در مطالعه خود نشان دادند آزمون

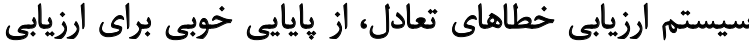

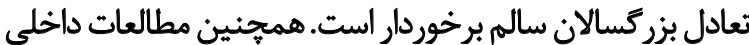

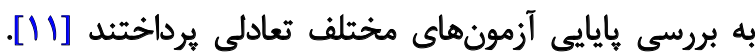

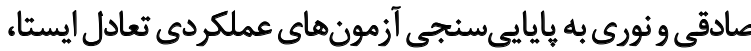

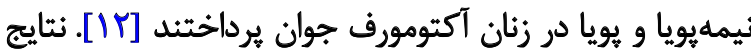

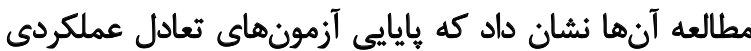

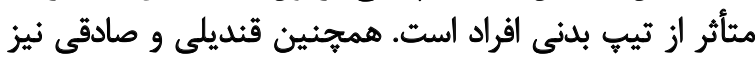

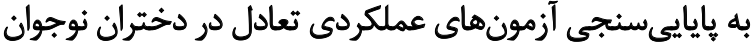

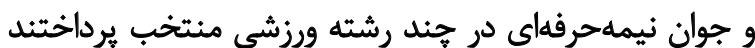

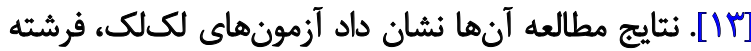

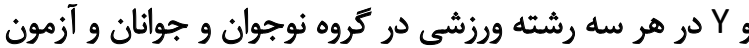

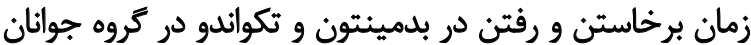

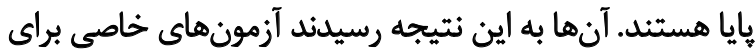

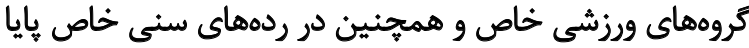

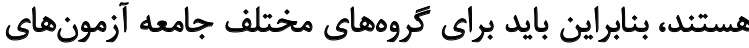

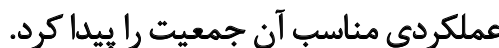

نيايايى يكى از ويرَّىهاى آزمونهاي استاندارد است؛ يعنى

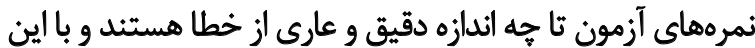

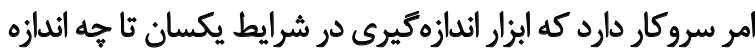

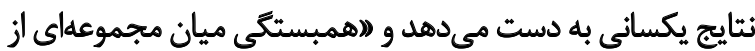

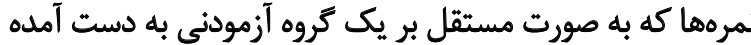

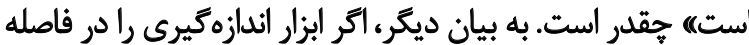

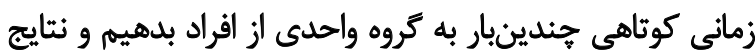

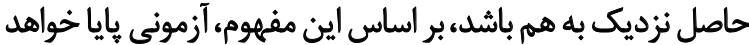

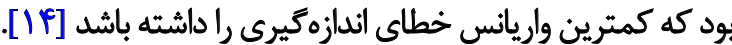
اندازه هاياييى باضريب همبستخى بيان مىشود و معيار كلى براي

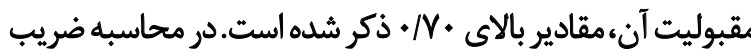

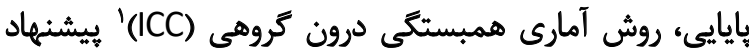

1. Intraclass Correlation Coefficient 


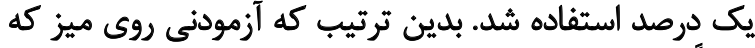

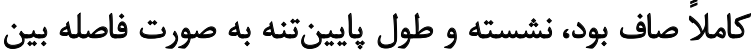

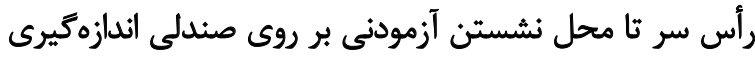

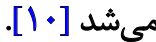

\section{نحوه اندازهييرى طول كف يا}

براي اندازهيرى طول كف يا هم از كوليس (مدل Guangla

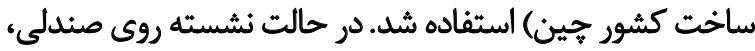

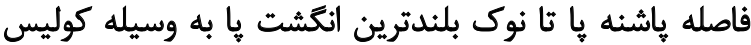

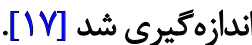

\section{نحوه اندازهيرى آزمون رومبرتى}

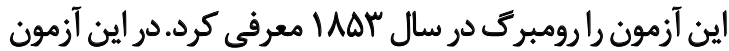

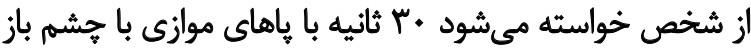

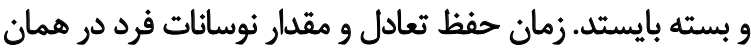

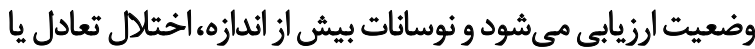

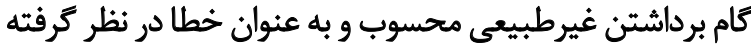

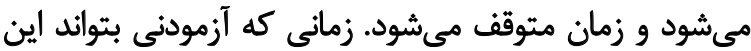

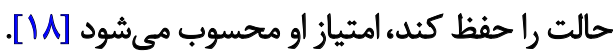

نحوه اندازميرى آزمون شاريند رومبرى؟

اين آزمون شامل وضعيت ثابتى است كه در آن آزمودنى بدون

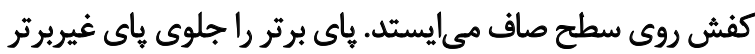

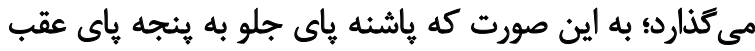

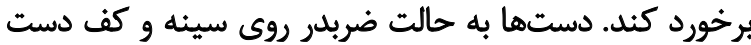

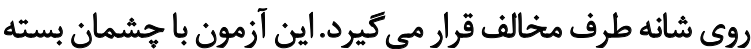

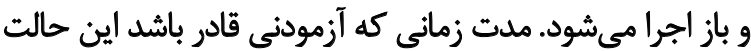

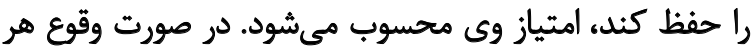

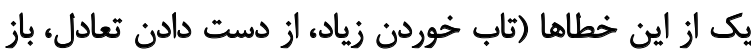

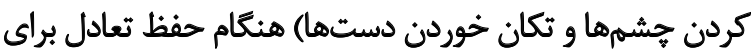

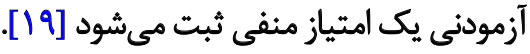

\section{نحوه اندازهيرى آزمون زمان برخاستن و راه رفتنج}

براى اجراى اين آزمون ابتدا يك صندلى بدون دستكيره به

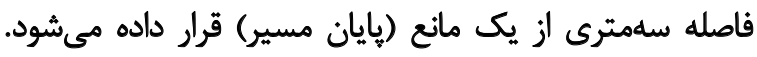

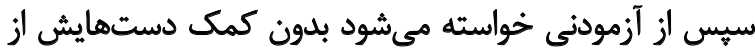

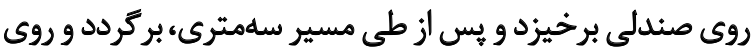

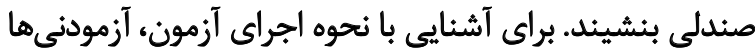

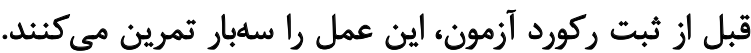

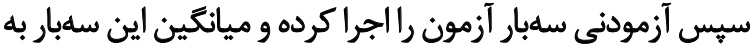

4. Romberg

5. Sharpened Romberg

6. Timed Get up \& Go
اطلاعات كامل در مورد يروتكل انجام كار و نحوه ئروهش و

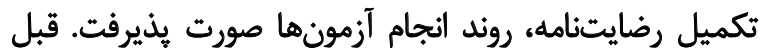

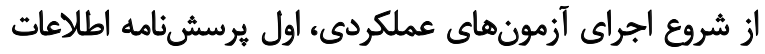

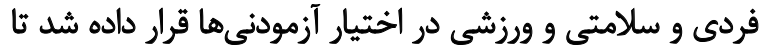

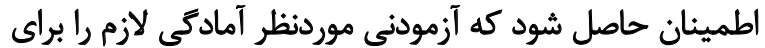

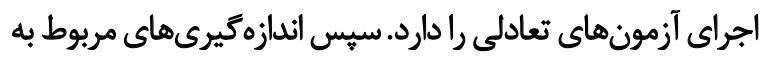

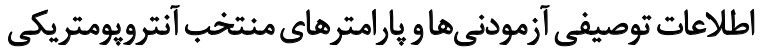

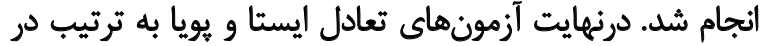

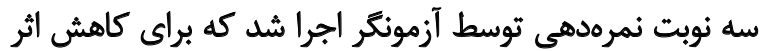

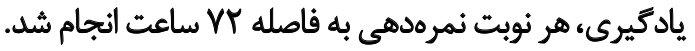

$$
\text { اندازٔكيرى فاكتور هاى مثتخب آتثرويومتريكى }
$$

نحوه اندازهيَيرى قد

قدسنج مدل سكاباضريب خطاي يك درصد، جهت اندازهيرى

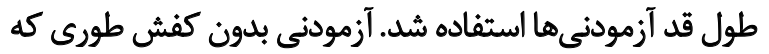

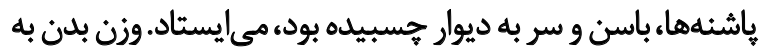

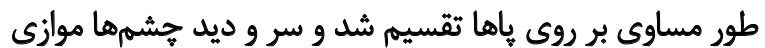

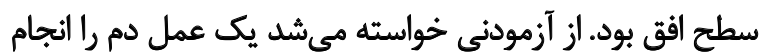

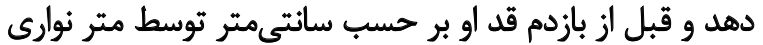

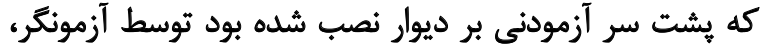

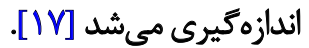

نحوه اندازهتيرى وزن

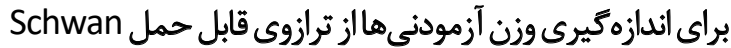

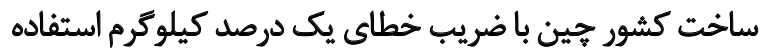

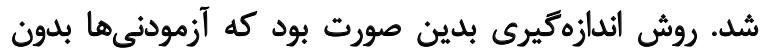

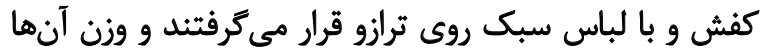

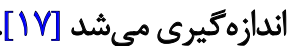

نحوه اندازهكيرى طول يايينتنه

براى اندازهيرى طول بايينتنه، از متر نوارى با بريب خطاى

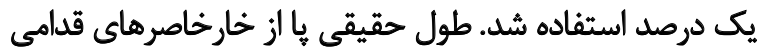

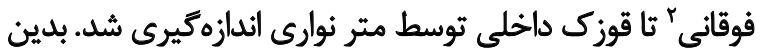

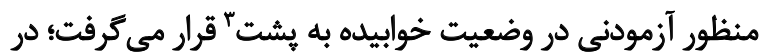

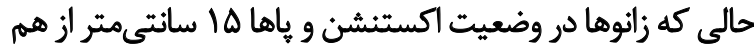

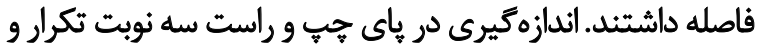

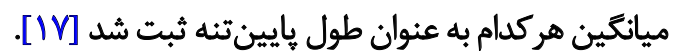
نحوه اندازميَي طول بالاتنه براى اندازهيرى طول بالاتنه، از متر نوارى با ضريب خطاى

2. Anterior Superior Iliac spine (ASIS)

3. Supine position 
خود را حفظ كند، آن عمل دستيابي مربوطه حذف واز آزمودنى

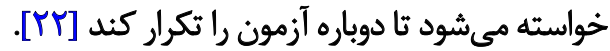

\section{روش ثجزيلوتحليل دادهها}

در اين تحقيق از آمار توصيفى براى توصيف دادههاى هر

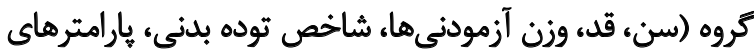

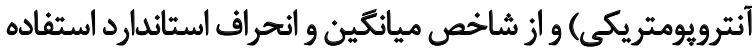

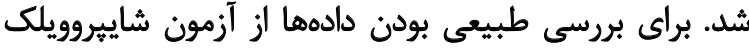

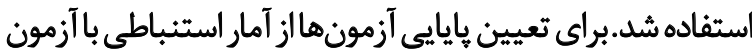

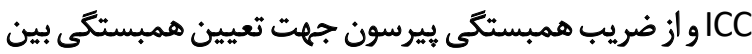

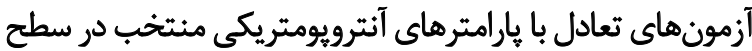

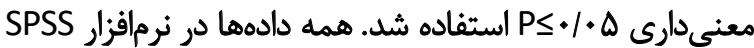
نسخه Y جمع آورى شد.

ثتايج

اطلاعات مربوط به ويزگكىهاى فردى آزمودنىها و يارامترهاى

آنترويومتريكى در جدول شماره ا نشان داده شده است أستون.

بر اساس يافتههاى حاصل از آزمون ICC در بروسى بإيايى

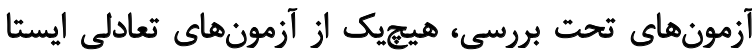

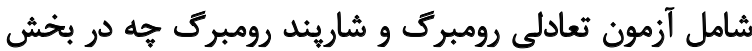

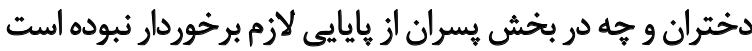

(جدول شماره Y).

بر اساس يافتههاى حاصل از آزمون ICC در بررسى پِايايى

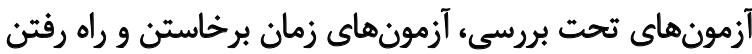
و آزمون راه رفتن ثاندوم (ICC=•/V9)

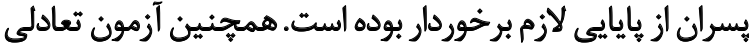

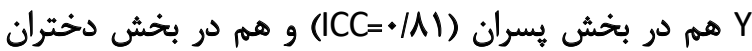
(ICC=•/A•)

نتايج حاصل از اين تحقيق در بررسى همبستگى بين إين

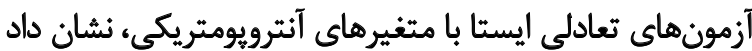

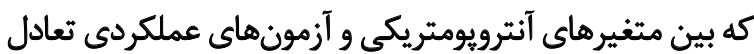

ايستا ارتباط معنادارى وجود ندارد (جدول شماره f).

نتايج حاصل از اين تحقيق در بررسى همبستّى بين آزمونهاي

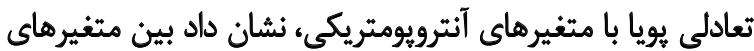

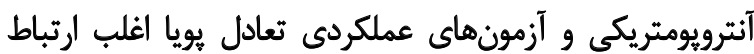

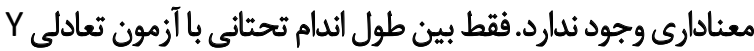

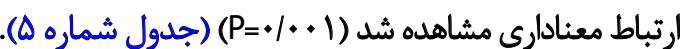

بحث

هدف از مطالعه حاضر بررسى يايايى إسنجى آزمون هاي عملكردي

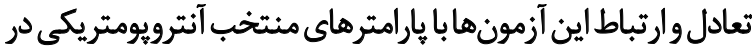

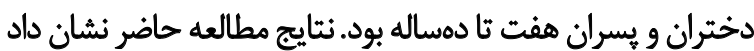

عنوان ركوردش ثبت مىشود [r·].

نحوه اندازهتيرى راه رفتن تاندوم

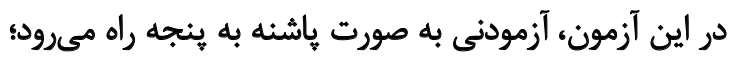

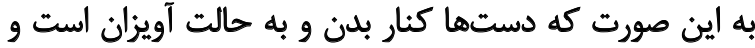

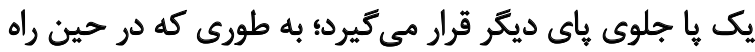

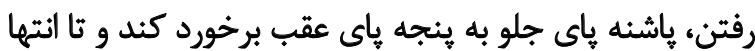

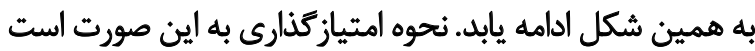

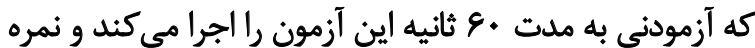

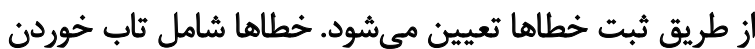

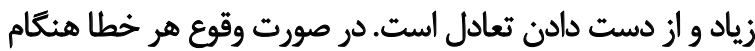

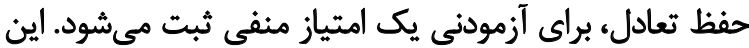

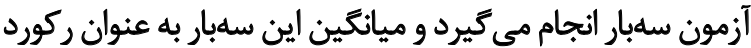

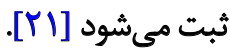

\section{نحوه اندازهيرى آزمون تعادلى نY}

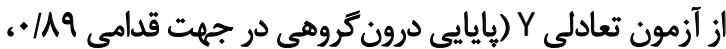

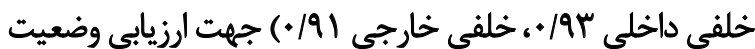

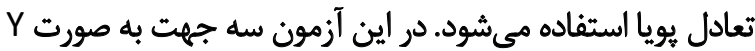

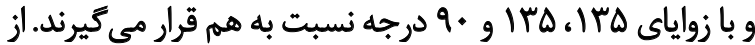

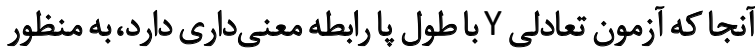

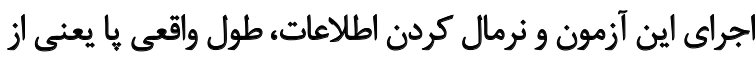

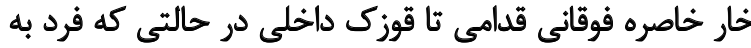

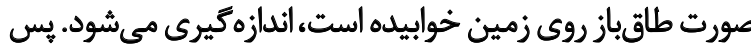

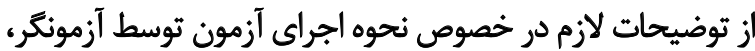

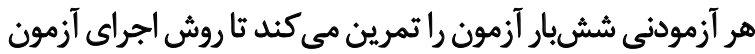

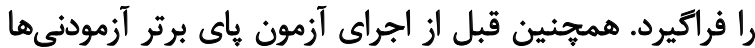

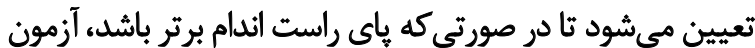

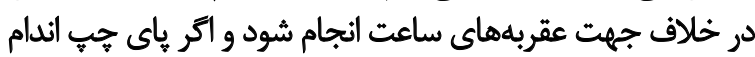
برتر باشد آزمون در جهت عقربهائهاي ساعت انجام شود.

آزمودنى در مركز Y قرار مى كيرد، روى يكى بيا مىايستد و باى

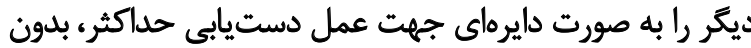

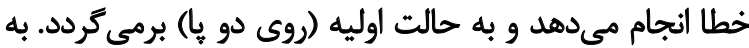

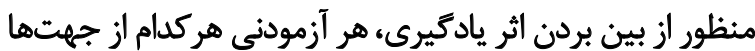

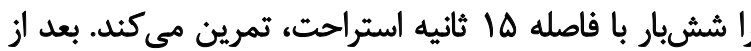

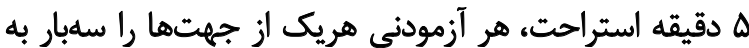

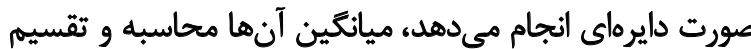

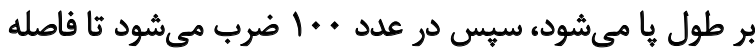

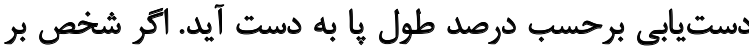

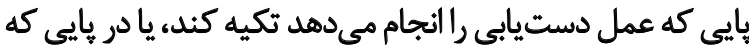

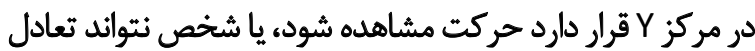

7. Tandem Walking test

8. Y Balance test 
جدول ا. ميانتين و انحراف استائدارد مشخصات فردى آزمودنى ها و يارامترهاي آنترويومتريكى

\begin{tabular}{|c|c|c|}
\hline 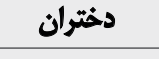 & | بسران & متغ \\
\hline \multicolumn{2}{|c|}{ ميانكين_|نحراف|ستاندارد } & مسير \\
\hline$N r E \pm 1 / r A$ & $N \Delta \cdot \pm / / M r$ & سن (سال) \\
\hline ITT/MINAF & ITV/THIIN·r & قد (سانتى هتر) \\
\hline$T W / \cdot \pm \Delta / 9 V$ & TNMYII./1. & جرم (كيلوكرم) \\
\hline TIRAAV/AT & $r \cdot \mid N E \pm 1 / q T$ & طول كف با (سانتىمت) \\
\hline$\Lambda \cdot / \pi) \pm \& / \pi q$ & W/ArEq/.q & طول يايينتثنه (سانتى متر) \\
\hline rq/rrm $\pm r / r+$ & rq/qE \pm r/ar & طول بالاتنه (سانتىمتر) \\
\hline$T r / T Y \pm T / F q$ & $r M / T \lambda \pm r / T r$ & شاخص توده بلن (كيلوكرم بر مترمربع) \\
\hline
\end{tabular}

مجله بيومكانيك ورنث

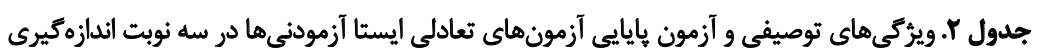

\begin{tabular}{|c|c|c|c|c|c|c|}
\hline \multicolumn{2}{|c|}{ بإيايى } & نوبت سوهم & نوبت دوم & ثويث أول & \multirow{2}{*}{ جنس } & \multirow{2}{*}{ آزهون } \\
\hline $\mathbf{P}$ & ICC & \multicolumn{3}{|c|}{ ميانئين ثانحراف استائدارو } & & \\
\hline.$/ 1 r$ & .181 & $\Delta \varphi / T A \pm I r / T V$ & a) $/ \lambda \Delta \pm 1 / / /$. & $F A / T E \pm I N A \Delta$ & يسران & رومبرى \\
\hline . & .119 & $\Delta Q / \Delta Y \pm 1 \cdot / Q V$ & $\Delta \& / T \cdot \pm N \Delta$. & $e q / n+ \pm 1 \% 1.8$ & دختران & ) (ثانيه) \\
\hline . &.$/ M P$ & $1 . / \Delta \Delta \pm r / \Delta r$ & $11 / N \cdot \pm \& / V^{P}$ & $\mid \varphi / K Y \pm \Delta / R T$ & يسران & شاريند رومبرى \\
\hline .111 & $\cdot / \Delta r$ & $W N \Delta \pm r / \Delta \mid$ & $r / m \pm V / r e$ & $r+/ 8 A \pm Y / M Y$ & دختران & (ثانيهان \\
\hline
\end{tabular}

مجله بيومكانيك ورنث

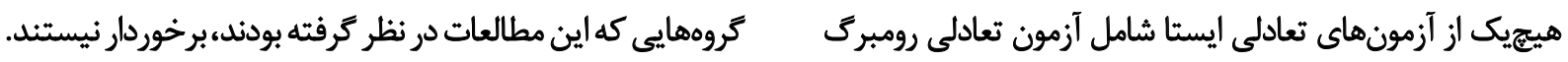

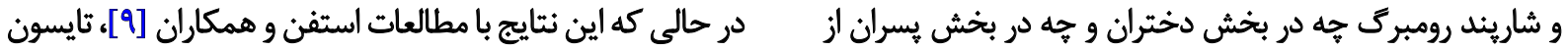

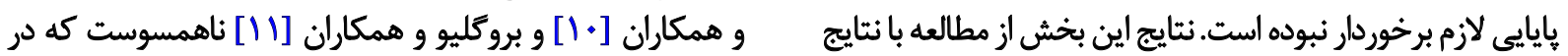

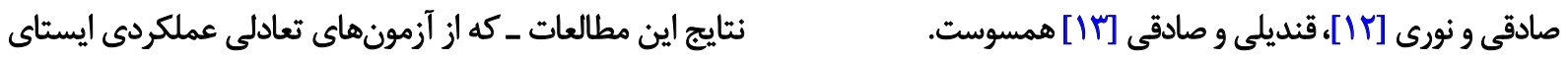

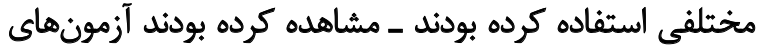

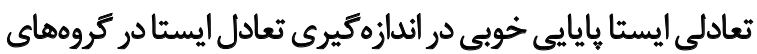
مختلف دارند.

در بخش نتايج اين مطالعات نيز مشخص شد تعدادى ازيازي

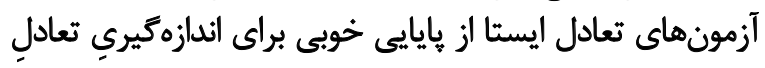

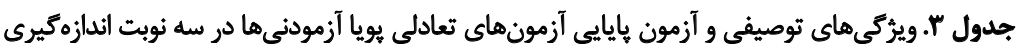

\begin{tabular}{|c|c|c|c|c|c|c|}
\hline \multicolumn{2}{|c|}{ بإيايى } & نويت سوم & نوبت دوم & نوبت اول & \multirow{2}{*}{ جنس } & \multirow{2}{*}{ آز أزمون } \\
\hline $\mathbf{P}$ & ICC & \multicolumn{3}{|c|}{ ميانئينثانحراف استاندارد } & & \\
\hline $\begin{array}{l}.1 \cdot 4 \\
.1+1\end{array}$ & $\begin{array}{l}.119 \\
.108\end{array}$ & $\begin{array}{l}\& / \Delta V \pm+/ \Delta r \\
V / \Delta V \pm \mid / Q V\end{array}$ & $\begin{array}{l}\& / M Y \pm / 81 \\
\Delta / V E \pm V / \Delta Y\end{array}$ & $\begin{array}{l}e / V A \pm / 9 \\
P / 9 \pm+/ 91\end{array}$ & نيسران & زمان برخاستن و راه رفتن (ثائيه) \\
\hline $\begin{array}{l}. / . r^{*} \\
. / 1 r\end{array}$ & $\begin{array}{l}. / N a \\
. / P A\end{array}$ & $\begin{array}{l}11 / r T \pm r / r A \\
1 . / 19 \pm \Delta / q 1\end{array}$ & $\begin{array}{l}\mid r / V \cdot \pm T / M^{e} \\
\mid Q / \| \pm E / T V\end{array}$ & $\begin{array}{l}1 W / r \mid \Psi / K A \\
\mid r / r r \pm T / M A\end{array}$ & نهتران & راه رفتن تأندوم (تعلفاد خطا) \\
\hline $\begin{array}{l}+1.1^{*} \\
+1.1^{*}\end{array}$ & $\begin{array}{l}\cdot|A| \\
\cdot \mid A .\end{array}$ & $\begin{array}{l}n / q \cdot \pm 11 / q \\
V \cdot / r r \pm I r / q V\end{array}$ & 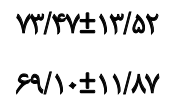 & $\begin{array}{l}Y / Q Y \pm \mid Y / \Delta 1 \\
Q Q / \Delta \Delta \pm \mid r / T 1\end{array}$ & دختران & آزمون Y (درصد طول عا) \\
\hline
\end{tabular}


جدول f. ضرايب همبستكى بين آزمونهاى عملكردى تعادل ايستا با بارامترهاى آنترويومتريكى آزمودنىهاى تحقيق

\begin{tabular}{|c|c|c|c|c|c|c|c|c|}
\hline \multicolumn{4}{|c|}{ شاريند رومبركى } & \multicolumn{4}{|c|}{ رومبركى } & \multirow{3}{*}{ بارامترهاى أنترويومتريكى } \\
\hline \multicolumn{2}{|c|}{ دختران } & \multicolumn{2}{|c|}{ بسران } & \multicolumn{2}{|c|}{ دختران } & \multicolumn{2}{|c|}{ بـران } & \\
\hline p & $r$ & $p$ & $r$ & $\mathbf{p}$ & $r$ & $p$ & $r$ & \\
\hline.$/ p q$ & זו/. & $\cdot / \pi \varphi$ & .119 & .1 .9 &.$- / K \varepsilon$ & / $/ \Delta T^{\prime}$ & .11 & سن (سال) \\
\hline.$/ M$ &.$/ 10$ & .190 & $\% v$ & $\% 1$ &.$- / T V$ & . I9 & $-.1 . v$ & جرم (كيلوكرم) \\
\hline.$/ 9$. & $.1+1$ &.$/ 11$ &.$/ T \Delta$ & $.1 \% \Delta$ &.$- / 41$ & $.10 \mathrm{~A}$ & $\% 1$ & قد (cm) \\
\hline.$/ \Delta 9$ & $\cdot 1 * 1$ &.$/ 4 \pi$ &.$/ N$ &.$/ 18$ &.$- / M T$ & $.1 \Delta F$ & -.1 .9 & طول كف با (cm) \\
\hline . &.$/ 10$ &.$/ \pi v$ &.$/ 1 F$ & Tr & -.119 & $\cdot N A$ &.+4 & طول بالاتنه (cm) \\
\hline. & $+1+1$ &.$/ 79$ & $-+1+4$ & 1.9 & $-+/ \pi Y$ & $\cdot / \Delta V$ & -+1.9 & طول بإيينتنه (cm) \\
\hline
\end{tabular}

مجلل بيومكانيك وزنش

جدول هـ ضرايب همبستى بين آزمونهاى عملكردى تعادل يويا با بارامترهاى آنترويومتريكى آزمودنىهاى تحقيق

\begin{tabular}{|c|c|c|c|c|c|c|c|c|c|c|c|c|}
\hline \multicolumn{4}{|c|}{ آزمون Y } & \multicolumn{4}{|c|}{ راه رفتن تاندوم } & \multicolumn{4}{|c|}{ زمان برخاستن و راه رفتن } & \multirow{3}{*}{ يار امترهاى آنترويومتريكى } \\
\hline \multicolumn{2}{|c|}{ 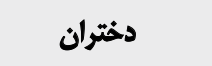 } & \multicolumn{2}{|c|}{ 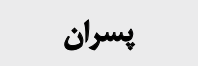 } & \multicolumn{2}{|c|}{ 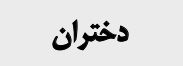 } & \multicolumn{2}{|c|}{ 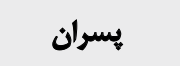 } & \multicolumn{2}{|c|}{ 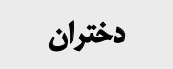 } & \multicolumn{2}{|c|}{ 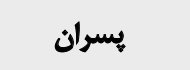 } & \\
\hline$p$ & $r$ & $p$ & $r$ & $p$ & $r$ & $p$ & $r$ & $p$ & $r$ & $p$ & $r$ & \\
\hline . /8T" & $-+/ 11$ &.$/ M Y$ & $* / 1 r$ &.$/ 94$ & $+1+1$ &.$/ F \Delta$ &.$- / 1 r$ &.$/ 90$ & .1 .9 & $.1 \& 8$ & $-+1+V$ & سن (سال) \\
\hline$\cdot / M$ & .1 .0 & $\cdot / r$ & $-\cdot / 1 f$ & $\cdot / 4 E$ &.$- / 11$ & $\cdot / M$ &.$- / 6 r$ & .181 & .1 .9 & $\cdot M$ & $-+1+4$ & جرم (كيلوكرم) \\
\hline$* / I r$ & .118 & .1 .9 & .1 .9 & .189 & -.1 .8 & .184 & $* / * V$ &.$/ I F$ & . &.$/ 91$ & .1 .1 & قد (cm) \\
\hline .18 &.$/ 1 \%$ & $\cdot / M$ & .1 .8 & - Mq & $-.1 . f^{f}$ &.$/ \% \Delta$ &.$/ 10$ &.$/ M A$ &.$/ 1 \mathrm{r}$ & . & -.118 & طول كف يا (cm) \\
\hline.$/ 1 F$ & $\cdot 1 \cdot r$ &.$/ \pi \Delta$ & .118 & .180 & $.1 \cdot v$ & .18 & $.1 * 1$ & 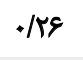 & $.1 \mathrm{~A}$ &.$/ \pi$ & $.11 f$ & طول بالاتئه (cm) \\
\hline $.10 .1^{*}$ & $\cdot 10 T$ & $.1 . .1$ &.$|8|$ & .198 & $.1 \cdot v$ &.$/ Q V$ & .1 .9 &.$/ M r$ & .119 & .180 & $-* / \cdot v$ & طول هاييينته (cm) \\
\hline
\end{tabular}

مجله بيومكانيك وزنش

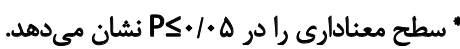

اين آزمونها مىتوائند از إيايايى خوبى در اندازمكيرى تعادل

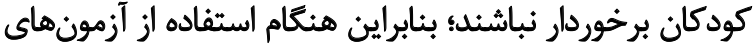

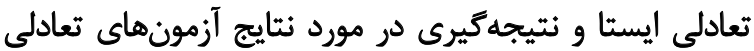

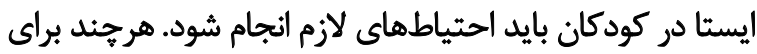

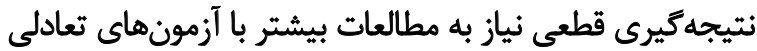
ايستاي مختلف است.

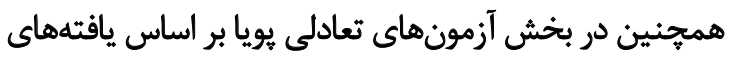

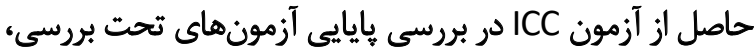

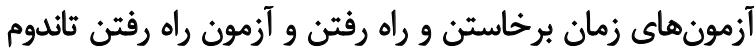

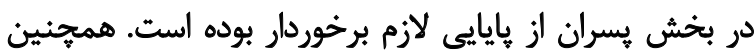

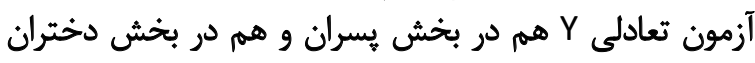

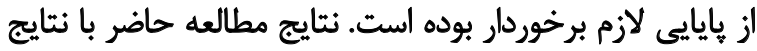

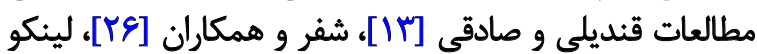

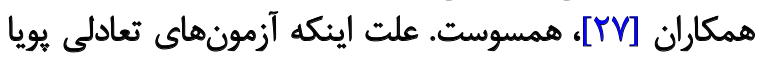

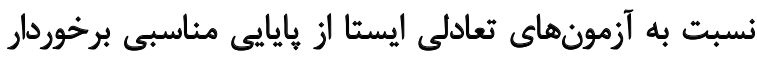

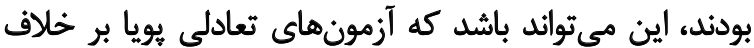

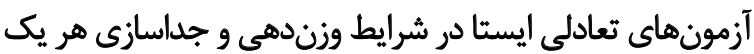

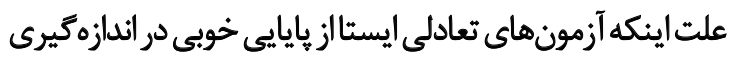

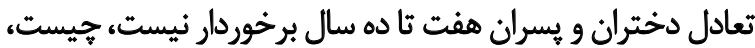

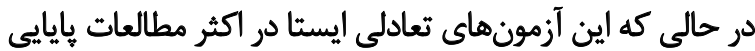

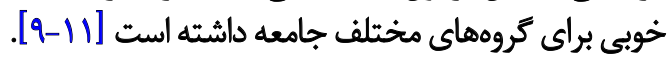

اطلاعات حسى متعددى از سرثاسر بدن براى حفظ تعادل

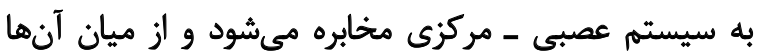

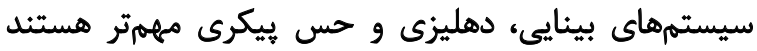

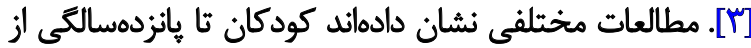

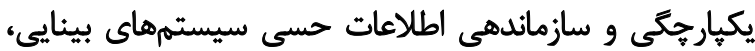

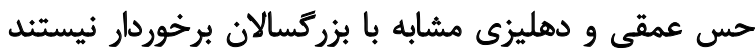

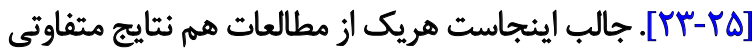

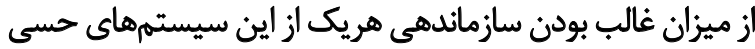

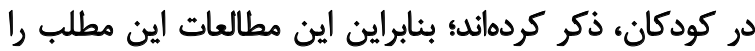

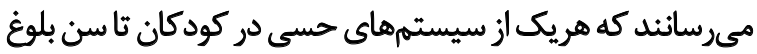

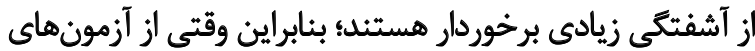

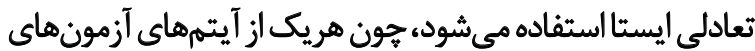

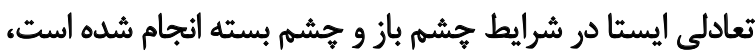


نكرفته است؛ هرجند براى نتيجه گيرى قطعى نياز به مطالعات بيشُترى است.

\section{نتيجليرى نهبايى}

در نتيجه كيرى اين مطالعه مي توان اين كونه تفسير كرد كه به به

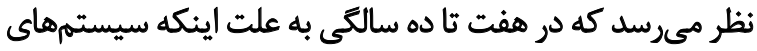

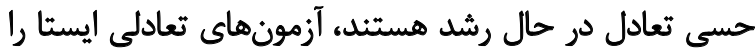

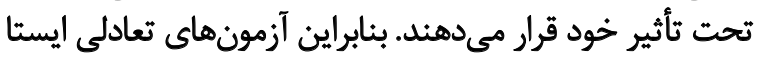

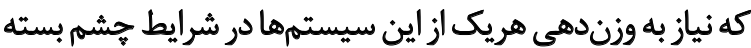

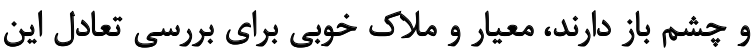

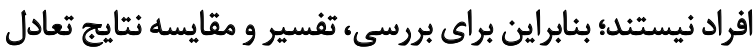

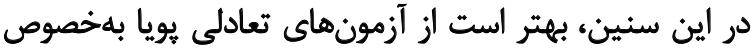
آزمون تعادلى Y استفاده شود.

ملاحظات اخلاقى ي ميروى از اصول اخلاق يثوهش

در اين مقاله كليه اصول اخلاقى در نظر كرفته شد.

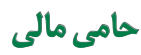

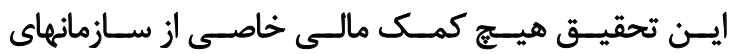

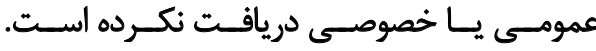

$$
\text { مشاركت نويسند }
$$

مفهومســازي، روششناسـى و نظـارت: تمامسى نويســندكان؛

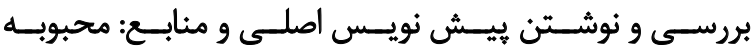

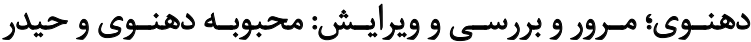

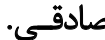
تعارض منافع بنابر اظهار نويسندكان اين مقاله تعارض منافع ندارد.

$$
\text { از سيستمهاى حسى صورت نكرفته است. }
$$

در حالى كه در اجراى آزمونهاى تعادلى ايستا نياز به تمركز

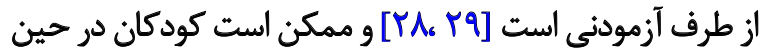

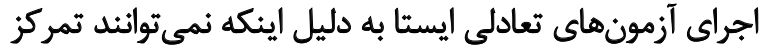

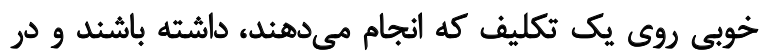

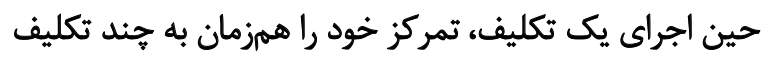

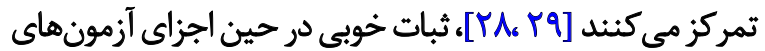

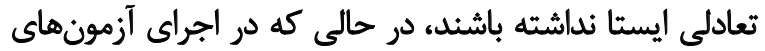

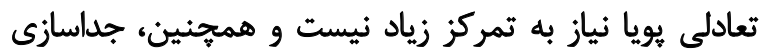

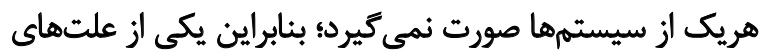

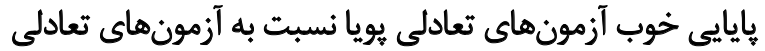
ايستا توضيحات ذكرشده، مى تواند باشد.

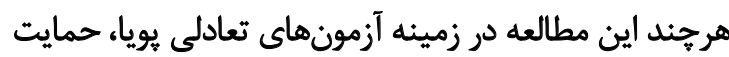

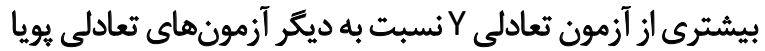

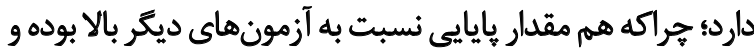

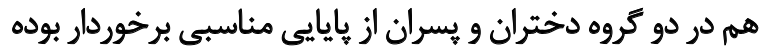

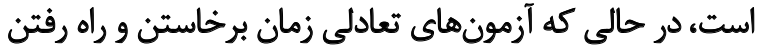

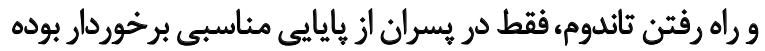

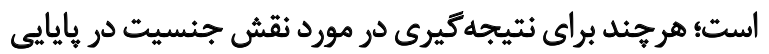

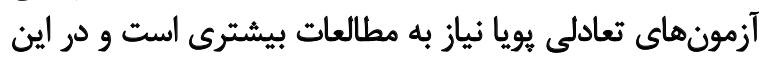

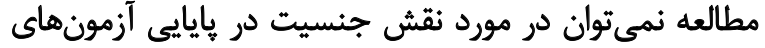

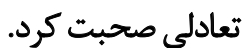

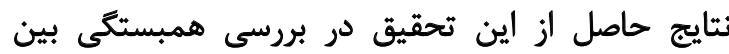

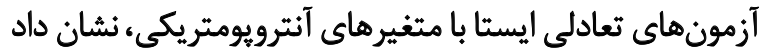

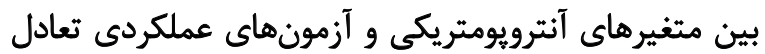

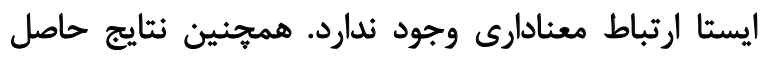

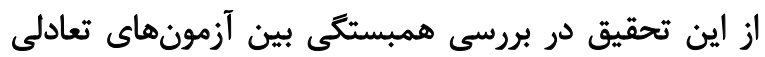

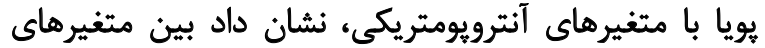

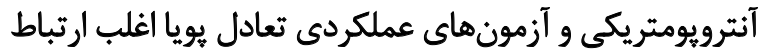

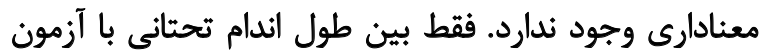

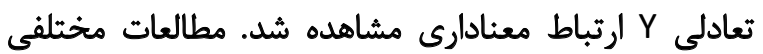

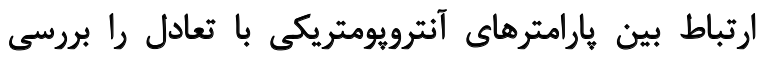

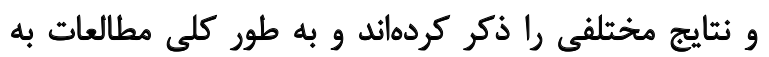

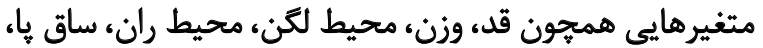
طول يايينتيه و طول پا را در ارتباط با تعادل اشاره كردهاند [r..rl]

ولى در مطالعه حاضر فقط ارتباط بين طول هايينتنه با بادي

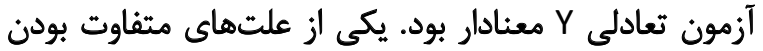

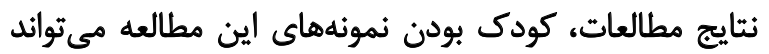

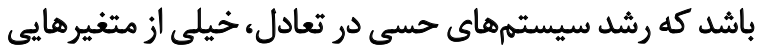

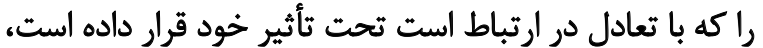

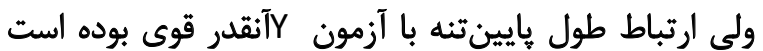

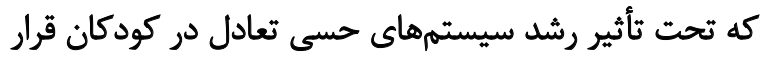




\section{References}

[1] Kamalian Lari S, Haghgoo HA, Farzad M, Hosseinzadeh S. [Investigation of the validity and reliability of Balance Evaluation Systems Test (BESTest) in assessment of balance disorders in people with multiple sclerosis (Persian)]. Arch Rehab. 2018; 18(4):288-95. [DOI:10.21859/ jrehab.18.4.3]

[2] Ivanenko Y, Gurfinkel VS. Human postural control. Front Neuroscie. 2018; 12:171. [DOI:10.3389/fnins.2018.00171]

[3] Sheehan DP, Katz L. The effects of a daily, 6-week exergaming curriculum on balance in fourth grade children. J Sport Health Sci. 2013; 2(3):131-7. [DOI:10.1016/j.jshs.2013.02.002]

[4] Haywood KM, Getchell N. Life span motor development. Human kinetics; 2019.

[5] Khasnis A, Gokula RM. Romberg's test. J Postgrad Med. 2003; 49(2):16972. [PMID]

[6] Gras LZ, Ganley KJ, Bosch PR, Mayer JE, Pohl PS. Convergent validity of the Sharpened Romberg. Phys Occup Ther Geriatr. 2017; 35(2):99-108. [DOI:10.1080/02703181.2017.1307897]

[7] Bohannon RW. Reference values for the timed up and go test: A descriptive meta-analysis. J Geriatr Phys Ther. 2006; 29(2):64-8. [DOI:10.1519/00139143-200608000-00004] [PMID]

[8] Cohen HS, Mulavara AP, Peters BT, Sangi-Haghpeykar H, Kung DH, Mosier DR, et al. Sharpening the tandem walking test for screening peripheral neuropathy. South Med J. 2013; 106(10):565-9. [DOI:10.1097/ SMJ.0000000000000009] [PMID] [PMCID]

[9] Plisky PJ, Gorman PP, Butler RJ, Kiesel KB, Underwood FB, Elkins B. The reliability of an instrumented device for measuring components of the star excursion balance test. N Am J Sports Phys Ther. 2009; 4(2):92-9. [PMID] [PMCID]

[10] Mony PK, Swaminathan S, Gajendran JK, Vaz M. Quality assurance for accuracy of anthropometric measurements in clinical and epidemiological studies:[Errare humanum est= to err is human].Indian J Community Med. 2016; 41(2):98-102. [DOI:10.4103/0970-0218.173499] [PMID] [PMCID]

[11] Sadeghi M, Mahdavi Nejad R. The effect of an 8-week selected theraband training on balance and motor performance in young wrestlers. J Sport Biomech. 2019; 5(1):28-37. [DOI:10.32598/biomechanics.5.1.3]

[12] Jafarnezhadgero A, HeshmatiZadeh S, Salahi-Movasagh S, Saki F. Patterns of muscle activity measurement in male students with forward head posture compared to healthy peers during running. J Sport Biomech. 2019; 5(1):38-49. [DOI:10.32598/biomechanics.5.1.4]

[13] Ringhof S, Stein T. Biomechanical assessment of dynamic balance: Specificity of different balance tests. Hum Mov Sci. 2018; 58:140-7. [DOI:10.1016/j.humov.2018.02.004] [PMID]

[14] Patikas D. Gait and balance. Comorbid conditions in individuals with Intellectual Disabilities. Berlin: Springer; 2015. p. 317-49. [DOI:10.1007/978-3-319-15437-4_11]

[15] Angyan L, Teczely T, Angyan Z. Factors affecting postural stability of healthy young adults. Acta Physiol Hung. 2007; 94(4):289-99. [DOI:10.1556/APhysiol.94.2007.4.1] [PMID]

[16] Steffen T, Seney M. Test-retest reliability and minimal detectable change on balance and ambulation tests, the 36-item short-form health survey, and the unified Parkinson disease rating scale in people with parkinsonism. Phys Ther. 2008; 88(6):733-46. [DOI:10.2522/ptj.20070214] [PMID]
[17] Tyson SF, DeSouza LH. Reliability and validity of functional balance tests post stroke. Clin Rehabil. 2004; 18(8):916-23. [DOI:10.1191/0269215504cr821oa] [PMID]

[18] Broglio SP, Zhu W, Sopiarz K, Park Y. Generalizability theory analysis of balance error scoring system reliability in healthy young adults. J Ath Train. 2009; 44(5):497-502. [DOI:10.4085/1062-6050-44.5.497] [PMID] [PMCID]

[19] Sadeghi H, Noori S. [Reliability of functional balance static, semi-dynamic and dynamic tests in Ectomorph women aged youth (Persian)]. JESM. 2015; 7(1):35-55. [DOI: 10.22059/JSMED.2015.53792]

[20] Ghandili S, Sadeghi H. [Reliability assessment of functional balance tests in semi-professional adolescent and young girls of several selected sport fields (Persian)]. JESM. 2017; 9(1):1-14. [DOI: 10.22059/ JSMED.2017.62868]

[21] Thomas JR, Nelson JK, Silverman SJ. Research methods in physical activity. Human kinetics; 2015.

[22] Bryman A. Social research methods. Oxford: Oxford university press; 2016.

[23] Cumberworth VL, Patel NN, Rogers W, Kenyon GS. The maturation of balance in children. J Laryngol Otol. 2007; 121(5):449-54. [DOI:10.1017/ S0022215106004051] [PMID]

[24] Hsu Y-S, Kuan C-C, Young Y-H. Assessing the development of balance function in children using stabilometry. Int J Pediatr Otorhinolaryngol. 2009; 73(5):737-40. [DOI:10.1016/j.ijporl.2009.01.016] [PMID]

[25] Steindl R, Kunz K, Schrott-Fischer A, Scholtz AW. Effect of age and sex on maturation of sensory systems and balance control. Dev Med Child Neurol. 2006; 48(6):477-82. [DOI:10.1111/j.1469-8749.2006.tb01299.x] [PMID]

[26] Shaffer SW, Teyhen DS, Lorenson CL, Warren RL, Koreerat CM, Straseske CA, et al. Y-balance test: A reliability study involving multiple raters. Mil Med. 2013; 178(11):1264-70. [DOI:10.7205/MILMED-D-13-00222] [PMID]

[27] Linek P, Sikora D, Wolny T, Saulicz E. Reliability and number of trials of Y Balance Test in adolescent athletes. Musculoskelet Sci Pract. 2017 31:72-5. [DOI:10.1016/j.msksp.2017.03.011] [PMID]

[28] Taghizadeh F, Dehghani F, Daneshfar A. The effect of focus of attention in imagery on the static balance of young girls. Sports Science. 2015; $8(2): 75-80$.

[29] Ricotti L. Static and dynamic balance in young athletes. JHSE. 2011; 6(4):616-28. [DOI:10.4100/jhse.2011.64.05]

[30] Greve JMDA, Cuğ M, Dülgeroğlu D, Brech GC, Alonso AC. Relationship between anthropometric factors, gender, and balance under unstable conditions in young adults. Biomed Res Int. 2013; 2013:850424. [DOI:10.1155/2013/850424] [PMID] [PMCID]

[31] Alonso AC, Luna NMS, Mochizuki L, Barbieri F, Santos S, Greve JMDA. The influence of anthropometric factors on postural balance: The relationship between body composition and posturographic measurements in young adults. Clinics. 2012; 67(12):1433-41. [DOI:10.6061/ clinics/2012(12)14] [PMID] [PMCID] 
This Page Intentionally Left Blank 